\title{
Fetomaternal alloimmunity as a cause of liver disease
}

\author{
Daniel Smyk • Tassos Grammatikopoulos • \\ Alexandros Daponte $\cdot$ Eirini I. Rigopoulou • \\ Dimitrios P. Bogdanos
}

Received: 1 February 2011/Accepted: 14 February 2011/Published online: 23 March 2011

(C) Springer-Verlag 2011

\begin{abstract}
Fetomaternal alloimmune disease has traditionally been associated with haematological disease such as fetomaternal alloimmune thrombocytopaenia and $\mathrm{Rh}$ haemolytic anaemia, but is now known to also be organ specific. Alloimmune membranous glomerulonephritis (AMG) is one of the most well understood organ-specific alloimmune diseases. Neonatal haemochromatosis $(\mathrm{NH})$ is a rare condition characterised by early liver failure in infants, with evidence suggesting that it is also alloimmune. Both AMG and $\mathrm{NH}$ appear to involve the passive transfer of alloantibodies to the fetus, which bind a specific alloantigen, fix complement and activate the terminal complement cascade. Although differences between AMG and $\mathrm{NH}$ are known, and evidence of the presence of antigen-specific alloantibodies in $\mathrm{NH}$ is still missing, we will use AMG as an
\end{abstract}

D. Smyk · D. P. Bogdanos ( $₫)$

Liver Immunopathology and Immunodiagnostics, Institute of Liver Studies and Liver Unit, King's College London School of Medicine at King's College Hospital,

Denmark Hill Campus, London SE5 9RS, UK

e-mail: dimitrios.bogdanos@kcl.ac.uk

URL: http://www.bogdanoslab.com

T. Grammatikopoulos

Paediatric Liver, GI and Nutrition Centre, King's College

London School of Medicine at King's College Hospital,

London SE5 9RS, UK

\author{
A. Daponte \\ Department of Obstetrics and Gynaecology, \\ University of Thessaly Medical School, \\ 41222 Larissa, Thessaly, Greece
}

E. I. Rigopoulou

Department of Medicine and Research Laboratory of Internal

Medicine, University of Thessaly Medical School,

41222 Larissa, Thessaly, Greece example of fetomaternal organ specific alloimmune disease, and critically compare this to other emerging evidence that indicates that $\mathrm{NH}$ is also alloimmune.

Keywords Alloimmune - Autoimmunity · Immunisation · Intravenous immunoglobulin · Placenta

\section{Introduction}

Fetomaternal alloimmunity arises when antigens expressed by the fetus and placenta are not expressed in the mother, resulting in maternal immunisation, production of $\mathrm{IgG}$ isotype antibodies against that antigen, and the passage of the $\operatorname{IgG}$ to the fetus [1-3]. These IgG class alloimmune antibodies bind to the antigen and initiate an immune response, which causes organ damage. Fetomaternal alloimmunity is more commonly associated with haematological conditions such as fetomaternal alloimmune thrombocytopaenia (FMAIT), or Rh haemolytic anaemia [1-3]. FMAIT arises when fetal platelet antigens inherited from the father, are not present in the mother, resulting in the maternal alloimmunisation against these antigens $[1,2]$. FMAIT presents a more complicated immunopathology than idiopathic thrombocytopenia (ITP), as it involves the immunobiology of the mother, fetus, and placenta as well as changes to the maternal immune system during pregnancy $[2,3]$. RhD haemolytic anaemia develops when an RhD-negative mother gives birth to an RhD-positive child [1]. At the time of delivery, the mother is sensitised by the transplacental passage of RhD-positive fetal red cells from the fetus. In subsequent pregnancies involving an $\mathrm{RhD}$ positive fetus, alloantibodies cross the placenta from the third month of pregnancy, and recognise the fetal red blood cells as their target antigen leading to their destruction [1]. 
It is now becoming apparent that fetomaternal alloimmunisation is not limited to haematological targets, but can also result in organ specific disease. The best studied organ specific target of fetomaternal alloimmune disease is antenatal membranous glomerulonephritis (AMG) $[4,5]$. This condition occurs when a mother contains a mutation in the metallomembrane endopeptidase (MME) gene, resulting in abnormal expression of the neutral endopeptidase protein (NEP) [6]. When a fetus and placenta contain the normal MME gene copy, and therefore the normal NEP, maternal alloimmunisation may occur, followed by the production of anti-NEP antibodies [4-6]. The IgG class of the anti-NEP antibodies is then passed to the fetus via the placenta, resulting in intraglomerular immune complex deposition and renal damage [4-6] (Table 1).

It is now emerging that the liver can also be a target for immune mediated attack, where transfer of maternal autoantibodies or alloantibodies in utero causes fetal liver damage [7-13]. Antimitochondrial antibodies (AMA), normally associated with primary biliary cirrhosis (PBC) $[12,14-16]$, has been associated with liver damage when transferred from the mother to the fetus [7].

Evidence is also pointing to alloimmunisation as the cause of neonatal haemochromatosis $(\mathrm{NH})$, a disease characterised by liver failure and extrahepatic siderosis in the newborn $[8-10,17,18]$. This review will compare a well defined organ specific alloimmune disease, AMG, with $\mathrm{NH}$ which is now believed to be alloimmune in a majority of cases.

\section{Alloimmunisation in renal disease: a model for organ specific alloimmunity}

Membranous glomerulonephritis is a major cause of chronic renal disease and nephrotic syndrome [4-6]. It is characterised by immune deposits on the outer aspects of the glomerular basement membrane [4-6]. Several candidate proteins have been investigated as the antigen involved, including megalin [19, 20], dipeptidyl-peptidase IV and NEP [21, 22]. The latter two are expressed in human podocytes, and are involved in the formation of immune deposits in the animal models of the disease [21, 22]. The first conclusive evidence that identified NEP as the antigen involved in AMG came from a study by Debiec et al. [4], who identified anti-NEP antibodies in the mother of an infant with membranous glomerulonephritis. NEP is an enzyme on the cell surface which is involved in signal inhibition (4). It is found not only in renal tissue, but also in liver, breast, lungs, and placental microvilli, as well as in urine and serum $[4,23]$. In the case report by Debiec et al. [4], pregnancy induced immunisation of the mother, followed by transplacental passage of antibodies directed against a renal antigen was initially suspected, due to the early presentation of renal failure in this infant. Immunofluorescence of the renal biopsy material from the infant showed IgG and C3 deposition in the subepithelium of all identified glomeruli [4]. Several serum samples from the mother were available for analysis, including a sample taken 7 months before a previous miscarriage, as well one

Table 1 Features of antenatal membranous glomerulonephritis (AMG) and neonatal hemochromatosis (NH) in support or against alloimmunity

\begin{tabular}{|c|c|c|}
\hline Disease feature & $\begin{array}{l}\text { Antenatal membranous glomerulonephritis } \\
\text { (AMG) }\end{array}$ & Neonatal haemochromatosis $(\mathrm{NH})$ \\
\hline Organ specific? & Yes: Renal & Yes: Fetal Liver \\
\hline Antigen identified? & $\begin{array}{l}\text { Yes: Neutral endopeptidase (NEP). } \\
\text { Subsequent maternal production } \\
\text { of anti-NEP antibodies }\end{array}$ & $\begin{array}{l}\text { Unidentified: Believed to be isolated } \\
\text { to the fetal liver. Some report an antigen } \\
\text { in the } 32 \mathrm{kDa} \text { range }\end{array}$ \\
\hline IgG present in affected organ? & $\begin{array}{l}\text { Yes: IgG1, 3, } 4 \text { identified but IgG1 and } 3 \\
\text { appear to be pathogenic; IgG4 associated } \\
\text { with subclinical disease. IgG colocalised } \\
\text { with the antigen }\end{array}$ & Yes: IgG identified on hepatocytes \\
\hline $\begin{array}{l}\text { Membrane attack complex (MAC) } \\
\text { demonstrated in affected organ? }\end{array}$ & $\begin{array}{l}\text { Yes: Heavy C5b-9 staining colocalised with } \\
\text { the NEP antigen and IgG }\end{array}$ & $\begin{array}{l}\text { Yes: Increased C } 5 b-9 \text { on hepatocytes } \\
\text { of NH patients. }\end{array}$ \\
\hline $\begin{array}{l}\text { Intravenous immunoglobulin } \\
\text { (IVIG) treatment successful? }\end{array}$ & $\begin{array}{l}\text { Yes: IVIG treatment reduces the maternal } \\
\text { titers of anti-NEP antibodies }\end{array}$ & $\begin{array}{l}\text { Yes: IVIG treatment in mothers from the } 18 \text { th } \\
\text { week in subsequent pregnancies greatly } \\
\text { reduces the disease severity and incidence }\end{array}$ \\
\hline Animal models? & $\begin{array}{l}\text { Yes: Rabbits injected with } \mathrm{IgG} \text { from mothers } \\
\text { with AMG affected children developed } \\
\text { renal disease similar to AMG }\end{array}$ & $\begin{array}{l}\text { Yes: Pregnant mice injected with IgG from } \\
\text { mothers with NH affected children has } \\
\text { increased rates of stillbirths, and fetus' } \\
\text { showed severe liver injury similar to that } \\
\text { seen in NH }\end{array}$ \\
\hline $\begin{array}{l}\text { Aetiology of maternal } \\
\text { immunisation identified? }\end{array}$ & $\begin{array}{l}\text { Yes: Maternal defect in the Metallomembrane } \\
\text { Endopeptidase (MME) gene, which encodes NEP }\end{array}$ & Unknown \\
\hline
\end{tabular}


from 3 months into the pregnancy in question. Additional samples were taken at 5 weeks, and 7 months after delivery [4]. These serum samples were incubated with kidney samples from human, rabbit, and rat, which was followed by indirect immunofluorescence. There was no reaction seen with sera taken 7 months before a previous miscarriage; maternal serum from 3 months gestation, as well as 5 weeks and 7 months after delivery, showed reaction on the brush borders and capillary walls of all kidney specimens [4]. Similar studies were conducted with the infant's serum, obtained at 13 and 40 days after birth. Serum from 13 days after birth did react with all kidney specimens, but there was no reaction seen with samples taken 40 days post birth [4]. This group conducted further studies to identify the antigen(s) involved. Protein extracts from human podocytes, rabbit renal cortex, and rat brush border were incubated with anti-neutral endopeptidase monoclonal antibody (anti-NEP mAb), maternal sera from 5 weeks post delivery, and control sera. Immunoblotts of the proteins incubated with anti-NEP $\mathrm{mAb}$, as well as those incubated with the maternal sera, both showed banding in the $90 \mathrm{kDa}$ range. There was no reaction with the control sera [4]. Immunoprecipitation studies showed NEP in the antigenic fraction bound to maternal $\mathrm{IgG}$, and in the unbound fraction of the controls [4]. As well, inhibition studies showed that $95 \%$ of the NEP enzyme activity was blocked by phosphoramidon and thiorphan, which are specific inhibitors of NEP [4]. Furthermore, IgG and NEP showed co-localisation on the infant's kidney biopsies, and rabbits injected with maternal $\mathrm{IgG}$ fraction developed renal disease which was clinically and histologically similar to AMG. This did not occur in rabbits injected with IgG from the father of the infant [4]. Finally, NEP expression was demonstrated to be absent in the mother, and present in the father, which indicated that the maternal exposure to fetal or placental NEP (which was paternally derived), resulted in her immunisation and production of anti-NEP antibodies [4]. This data provided convincing evidence that the AMG in the infant was due to the passage of maternal anti-NEP antibodies. These antibodies were of the IgG class, which are the only antibodies that are capable of crossing the placenta, which begins between 17 and 22 weeks gestation [24]. Additionally, the gradually declining levels of the IgG in the infant would also suggest that they were of maternal origin, with the maternal exposure likely occurring at the time of her previous miscarriage [4], as anti-NEP antibodies were not present prior to this.

Ronco et al. [25] proposes several mechanisms by which fetomaternal alloimmunisation may occur. Fetal factors include the presence of an antigen that is only expressed in fetal life, and the fetal expression of a dominant paternal allele which differs from a maternal allovariant $[25,26]$. A genetic defect in the mother, resulting in the absence of a particular antigen that is expressed normally in the fetus, has been demonstrated in AMG [6, 26, 27]. Whatever the mechanism, a fetal antigen must be capable of passing the placenta into the maternal circulation causing alloimmunisation, followed by maternal production of antibodies against this antigen $[1-3,25]$. When maternal $\operatorname{IgG}$ is transferred to the fetus in weeks 17-22 gestation [24], an antibody-antigen reaction may occur, resulting in organ damage [1-3, 7, 25]. Debiec et al. [6] examined three families with histories of AMG, and found two heterozygous or homozygous truncating mutations in the MME gene (the gene encoding NEP) of five mothers. These mutations resulted in a premature stop codon, leading to the absence of NEP in the mothers [6]. Further studies of the anti-NEP IgG subclasses present in the glomeruli of AMG infants showed a predominance of IgG1 and IgG4 [6, 26]. The subclass of IgG involved also appeared to have an effect on the disease severity, with IgG1 producing clinical disease, and IgG4 producing no disease, or disease which was quiescent $[23,26,28]$. This may be due to IgG1 and IgG3 being stronger activators of the complement cascade, compared to IgG4, which is a weak activator [28, 29]. Complement has been found to co-localise with NEP and IgG in renal biopsies from AMG patients, as demonstrated by C5b-9 deposition in the glomeruli $[6,26,30]$. As AMG is clearly caused by maternal anti-NEP antibodies, Debiec and Ronco [26] advises the weekly administration of intravenous immunoglobulin (IVIG) and high dose corticosteroids by the 18 th week, in subsequent pregnancies of women with previous children affected by AMG. This is based on observation by that group, but there has been no cohort study examining the efficacy of IVIG treatment in AMG. One case report indicates that IVIG reduced the maternal anti-NEP antibodies titers by $30 \%$, and that plasma exchange reduced these titers by 50\% [23] (Fig. 1).

Alloimmune membranous glomerulonephritis provides us with an excellent model for organ specific fetomaternal alloimmunity. It is a condition that was very suggestive as having an alloimmune aetiology, but lacked an identifiable antigen. Continued research eventually identified the antigen as NEP [4]. The maternal defect of the MME gene causes the production of anti-NEP IgG when exposure to fetal and placental NEP occurs [4, 6]. Further elucidation of the immunopathogenesis showed that complement plays a role in the renal damage seen in many AMG patients [6]. This model may apply to liver specific diseases that appear to be a result of maternal antibodies, such as NH [9], where the antigen in question remains unidentified, and demonstration that the disease is caused by the maternal transfer of antibodies is lacking. There are, however, some case reports of liver disease in the neonatal period, which appears to be the result of an identified maternal autoantibody [7]. 


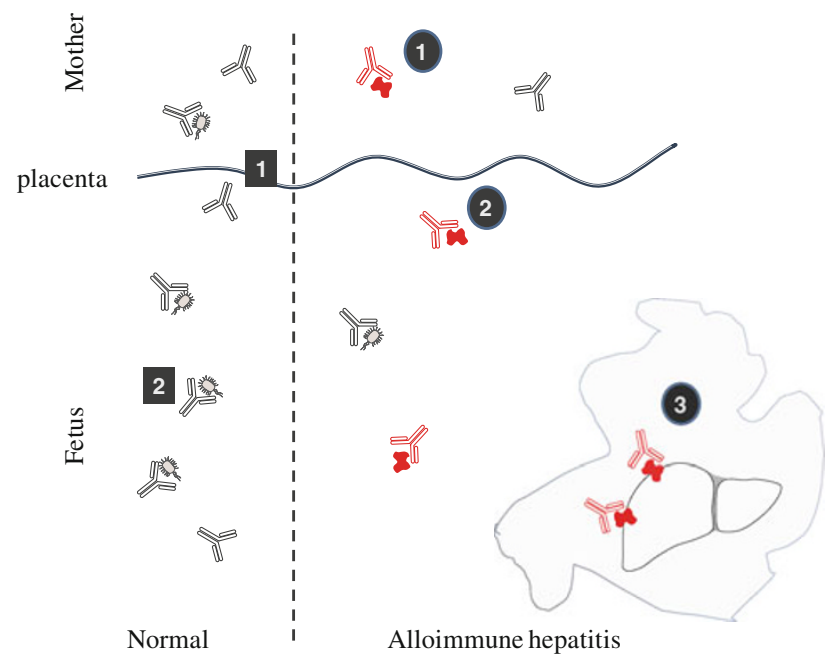

Fig. 1 Schematic drawing of the normal (left) versus immunopathogenic (right) transfer of antibodies from the mother to the fetus. Normally, maternal antibodies of the IgG class cross the placenta (box 1) from 17 to 22 week's gestational age. This passive transfer of immunity does not initiate an immune response by the maternal antibodies towards fetal antigens, but provides the fetus with humoral immunity (box 2) to protect against infectious agents. The alloimmune hypothesis behind neonatal haemochromatosis $(\mathrm{NH})$ involves the maternal exposure to a yet unknown fetal liver antigen, followed by the production of antibodies of the $\mathrm{IgG}$ class towards this antigen (circle 1). These antigens then cross the placenta, and enter fetal circulation (circle 2). Binding of these antibodies to the antigen on the fetal liver (circle 3) activates the terminal complement cascade, and the assembly of membrane attack complex, which causes hepatocyte lysis. This liver damage may appear as an acute hepatitis in some $\mathrm{NH}$ cases, or more commonly as a chronic/subacute hepatitis

\section{Antimitochondrial antibodies and neonatal liver disease}

Antimitochondrial antibodies are the serological hallmark of PBC, a disease occurring mostly in women in the fourth and fifth decade [7-13, 31-33]. AMA are directed against members of the 2-oxo-acid dehydrogenase complex of enzymes and the presence of these antigen-specific autoantibodies predicts the development of the disease in asymptomatic patients with normal liver biochemistry profiles [14, 32, 34, 35]. Within the paediatric population, AMA positivity is extremely rare, with some case reports associating liver disease with AMA in older children [3641]. In these reports, it is unlikely that the AMA was of maternal origin as maternal $\mathrm{IgG}$ is not present beyond the tenth month of life. Hannam et al. [7] reports the association of neonatal liver disease with the placental transfer of AMA in two infants. Whether AMA have the ability to induce biliary epithelial cell destruction, or whether they are an epiphenomena of bile duct damage is unclear [16, 42-46]. Also, the mechanisms responsible for the development of disease-specific mitochondrial immune responses are not well understood [47-50]. Several possibilities including xenobiotic induced autoimmunity or microbial/ self molecular mimicry and immunological crossreactivity have been suggested [12, 13, 31, 51-63].

Amongst the AMA-positive neonates described by Hannam et al. [7], the first was that of a female born to a mother with AMA positivity, who had normal liver function tests and was asymptomatic. The fetus was found to be hydropic in the 21 st week of pregnancy, transfused, and delivered at 31 weeks gestation with jaundice and deranged liver function tests [7]. The second infant was a term male, who presented at 5 weeks with poor weight gain, pale stools, and erythematous rash [7]. His liver function tests were deranged, and a liver biopsy showed cholestasis with hepatitis, mild cholangiolitic changes and portal fibrosis [7]. Infection, enzyme abnormalities, and $\mathrm{Rh} / \mathrm{ABO}$ incomparability were ruled out as a cause of the liver derangement in both infants, but both were found to be positive for AMA. Further studies of the AMA in both mother and child pairs showed that the AMA immunofluorescence pattern was of the M2 subtype (the subtype found in $\mathrm{PBC}$ ), and that the $\mathrm{IgG}$ subclasses were predominantly of the $\operatorname{IgG} 1$ followed by $\operatorname{IgG} 3$ subclass in both mother and child pairs [7]. IgG3 and IgG1 are the most predominant subclasses of AMA in patients with $\mathrm{PBC}$ [12, 54, 64]. Immunofluorescence of the children's liver biopsies showed peri-hepatocyte deposition of IgG. Epitope reactivity was predominantly for PDC-E3, and ILD PDC-E2 in both mother and child pairs, which is also found in PBC [7]. Although the children only had antibodies of the IgG class, the mothers also contained antibodies of the $\operatorname{IgA}$ and IgM classes, which is a feature frequently found in patients with PBC. The fact that the antibodies in the infants were $\mathrm{IgG}$, and that they were undetectable by 3-month follow up, suggested that the antibodies were of maternal origin [7]. Although it was not definitively determined that the liver disease in the two infants was caused by AMA, the fact that all other causes were ruled out, and that AMA is pathognomonic for liver disease, suggests that the AMA may have played a role in the liver disease seen in these two infants. AMA has been considered pathogenically relevant as asymptomatic seropositivity to AMA long before the development of full-blown disease is a common observation [14, 65, 66]. For antibodies with a pathogenic potential, complement-dependent and/or antibody-dependent cell-mediated cytotoxicity (ADCC) are the likely effectors of damage [12]. Recent studies suggest that disease-specific AMA-antigen complexes can be internalised, processed, and then presented by antigen-presenting cells (APCs) to induce specific cytotoxic T lymphocytes [44, 47, 50, 67]. It has also been shown that AMA can gain access to apoptotic cells with intact antigen, and that this interaction facilitates the perpetuation of the ongoing immune-mediated damage [ 68 , 
69]. Whether maternally transferred autoantibodies and/or fetomaternal alloantibodies directed against liver-specific antigens have pathogenic properties remains to be seen. An increasing number of studies have implicated fetomaternal alloimmunity as a cause of liver injury in $\mathrm{NH}$.

\section{NH: an alloimmune liver disease?}

Neonatal haemochromatosis is characterised by severe liver injury originating in utero, which is often accompanied by extrahepatic siderosis sparring the reticuloendothelial system [70-75]. The recurrence rate of $60-80 \%$ in pregnancies after the index case, as well as the fact that there is no apparent inheritance paternally has lead to the hypothesis that $\mathrm{NH}$ is an alloimmune disease [9]. Much like AMG, NH differs from alloimmune conditions in that it is organ specific. As previously mentioned, maternal $\operatorname{IgG}$ is passed to the fetus between the 17th and 22nd week gestational age [24], which has been suggested to correlate with the onset of liver injury in $\mathrm{NH}$ affected fetuses [76]. Investigations in regards to the alloimmune pathogenesis of $\mathrm{NH}$ follow a similar pattern to the studies of AMG performed by Debiec et al. [4]. Whittington and Malladi attempted to identify an $\mathrm{NH}$ specific antigen in fetal liver by immunoblotting proteins from different human fetal tissues with $\operatorname{IgG}$ from sera of mothers with $\mathrm{NH}$ affected infants, followed by sodium dodecyl sulfate polyacrylamide gel electrophoresis analysis [9]. A single band in the $32 \mathrm{kDa}$ range was found with the fetal liver tissue at 20 weeks gestation; no reaction was found with control liver samples from adults or children [9]. Fetal mouse liver at 10 weeks gestation and adult mouse liver were also tested; the only reaction was with the fetal mouse liver, again with a protein of $32 \mathrm{kDa}$ [9]. This $32 \mathrm{kDa}$ protein remains unidentified, and no further studies have been performed to elucidate its identity, or prevalence in $\mathrm{NH}$ cases. It does appear that the antigen involved in $\mathrm{NH}$ is only present or only expressed in fetal life [9]. It may also be expressed in the placenta if the immunisation of the mother occurred due to a genetic deficiency, where the fetus and placenta normally express the paternally derived protein, as seen with NEP in AMG [26].

A mouse model for $\mathrm{NH}$ has also been explored, where pregnant mice were injected with purified $\mathrm{IgG}$ from mothers with previous $\mathrm{NH}$ affected pregnancies, and controls were injected with non-NH sera or saline [77]. Mice injected with $\mathrm{NH}-\mathrm{IgG}$ showed an increased rate of stillbirth, with extensive fetal liver damage and lobular inflammation [77]. Controls showed normal litter sizes and no fetal liver injury [77]. Additionally, IgG applied to fetal mouse hepatocytes in the presence of complement showed extensive hepatocyte binding and lysis, which was not found with non-NH control $\operatorname{IgG}$ [77]. Debiec et al. [4] demonstrated that the $\operatorname{IgG}$ bound to glomeruli of AMG patients was primarily of the $\mathrm{IgG} 1$ and $\mathrm{IgG} 3$ class when the disease was symptomatic, and that complement had been activated. These findings have also been reproduced in the case of $\mathrm{NH}$, which suggests that maternal IgG1 and/or IgG3 binds to fetal hepatocyte cell surface antigens resulting in a terminal complement cascade (TCC), which leads to membrane attack complex (MAC)-mediated hepatocyte lysis [78]. Liver samples from 33 cases of confirmed NH were obtained; samples from 37 non-NH cases with severe liver disease and 11 infants who died of perinatal asphyxia were included as controls [78]. These specimens were immunostained with the monoclonal antibody to SC5b-9, which would stain positive in the presence of complement [78]. NH hepatocytes showed extensive positive staining, suggesting the assembly of MAC on the hepatocyte cell surface; the non-NH cases showed minimal positive staining, and controls from the asphyxiated infants showed no positive staining [78].

Although Pan et al. [78] provides compelling evidence that the classical pathway of complement is activated on $\mathrm{NH}$ hepatocytes, confirmatory testing should be performed to ensure that it is indeed the classical pathway activated [79]. This can be done by measuring $\mathrm{C} 4 \mathrm{~d}$ in biological fluids or tissues, as well as excluding the alternative pathway activation by measuring the fragment $\mathrm{Ba}$ [79]. Perhaps the most critical step in determining whether NH is truly alloimmune is the determination of the identity of the antigen(s) in question, as its lack of identity limits speculation as to whether $\mathrm{NH}$ is alloimmune or autoimmune [79].

As Debiec and Ronco [26] indicate IVIG treatment during pregnancy in mothers with previous AMG pregnancies, IVIG is also indicated in the same manner in $\mathrm{NH}$ $[17,18]$. Several cases report that IVIG treatment administered at a dose of $1 \mathrm{~g} / \mathrm{kg}$ per week from the 18th week to birth is successful in dramatically reducing the disease severity in subsequent pregnancies $[17,18,80,81]$. Whittington and Kelly demonstrated that 52 of 55 infants born to mothers treated with IVIG, all of which had previous histories of $\mathrm{NH}$ affected children, showed either no overt liver dysfunction, or mild liver dysfunction that was treated with supportive medical therapy [18]. The usefulness of IVIG treatment in these conditions is further evidence as to their alloimmune/autoimmune aetiology [18].

Whether $\mathrm{NH}$ is a true fetomaternal alloimmune disease remains to be seen $[9,79]$. The identification of an antigen that can serve as a target of the presumed alloimmune response is the prerequisite for $\mathrm{NH}$ to be classified as alloimmune. This will also allow for the detection of specific alloantibodies, as well as defining the genetic basis of the maternal alloimmunisation. These findings will 
contribute to better detection, and hence better management of those pregnancies which are at risk for developing $\mathrm{NH}$.

\section{Conclusion}

Fetomaternal alloimmune disease is not limited to the haematological system, but can also be organ specific, which has been well defined by AMG. Current evidence suggests that NH may also be an organ specific alloimmune disease in a majority of cases. Both AMG and NH share inheritance patterns similar to other alloimmune diseases. Sera from mothers of children with AMG and NH react with antigenic targets on the affected organ, and IgG has been identified on these target tissues, which can only be of maternal origin in infants. Immunoglobulins of the $\operatorname{IgG} 1$ and IgG3 class strongly fix complement, and the presence of C5b-9 has been demonstrated to be of high proportion in the affected tissues in AMG and NH. This is indicative that the TCC is activated, and plays a role in both conditions. Finally, the apparent success of IVIG treatments in both $\mathrm{AMG}$ and $\mathrm{NH}$ serve as further support of their alloimmune aetiology.

Current evidence suggests that NH may also be an organ specific alloimmune disease in a majority of cases, but the lack of an identifiable antigen and alloantibody prohibits this conclusion at the present time. More research is needed to elucidate the identity of the antigen(s) and alloantibody, as well as the underlying mechanisms responsible for the induction of this enigmatic disease.

Conflict of interest None.

\section{References}

1. Urbaniak SJ, Greiss MA (2000) RhD haemolytic disease of the fetus and the newborn. Blood Rev 14(1):44-61

2. Kaplan C (2002) Alloimmune thrombocytopenia of the fetus and the newborn. Blood Rev 16(1):69-72

3. Rozman P (2002) Platelet antigens. The role of human platelet alloantigens (HPA) in blood transfusion and transplantation. Transpl Immunol 10(2-3):165-181

4. Debiec H, Guigonis V, Mougenot B et al (2002) Antenatal membranous glomerulonephritis due to anti-neutral endopeptidase antibodies. N Engl J Med 346(26):2053-2060

5. Debiec H, Guigonis V, Mougenot B et al (2003) Antenatal membranous glomerulonephritis with vascular injury induced by anti-neutral endopeptidase antibodies: toward new concepts in the pathogenesis of glomerular diseases. J Am Soc Nephrol 14(Suppl 1):S27-S32

6. Debiec H, Nauta J, Coulet F et al (2004) Role of truncating mutations in MME gene in fetomaternal alloimmunisation and antenatal glomerulopathies. Lancet 364(9441):1252-1259

7. Hannam S, Bogdanos DP, Davies ET et al (2002) Neonatal liver disease associated with placental transfer of anti-mitochondrial antibodies. Autoimmunity 35(8):545-550
8. Whitington PF, Kelly S, Ekong UD (2005) Neonatal hemochromatosis: fetal liver disease leading to liver failure in the fetus and newborn. Pediatr Transplant 9(5):640-645

9. Whitington PF, Malladi P (2005) Neonatal hemochromatosis: is it an alloimmune disease? J Pediatr Gastroenterol Nutr 40(5): 544-549

10. Whitington PF (2007) Neonatal hemochromatosis: a congenital alloimmune hepatitis. Semin Liver Dis 27(3):243-250

11. Bernal W, Meda F, Ma Y et al (2008) Disease-specific autoantibodies in patients with acute liver failure: the King's College London Experience. Hepatology 47(3):1096-1097 (author reply 1097)

12. Bogdanos DP, Baum H, Vergani D (2003) Antimitochondrial and other autoantibodies. Clin Liver Dis 7(4):759-777, vi

13. Bogdanos DP, Mieli-Vergani G, Vergani D (2009) Autoantibodies and their antigens in autoimmune hepatitis. Semin Liver Dis 29(3):241-253

14. Kaplan MM, Gershwin ME (2005) Primary biliary cirrhosis. N Engl J Med 353(12):1261-1273

15. Invernizzi P, Lleo A, Podda M (2007) Interpreting serological tests in diagnosing autoimmune liver diseases. Semin Liver Dis 27(2):161-172

16. Liaskos C, Bogdanos DP, Rigopoulou EI et al (2007) Development of antimitochondrial antibodies in patients with autoimmune hepatitis: art of facts or an artifact? J Gastroenterol Hepatol 22(3):454-455

17. Whitington PF, Hibbard JU (2004) High-dose immunoglobulin during pregnancy for recurrent neonatal haemochromatosis. Lancet 364(9446): 1690-1698

18. Whitington PF, Kelly S (2008) Outcome of pregnancies at risk for neonatal hemochromatosis is improved by treatment with high-dose intravenous immunoglobulin. Pediatrics 121(6):e1615e1621

19. Kerjaschki D, Farquhar MG (1983) Immunocytochemical localization of the Heymann nephritis antigen (GP330) in glomerular epithelial cells of normal Lewis rats. J Exp Med 157(2):667-686

20. Saito A, Pietromonaco S, Loo AK et al (1994) Complete cloning and sequencing of rat gp330/"megalin", a distinctive member of the low density lipoprotein receptor gene family. Proc Natl Acad Sci USA 91(21):9725-9729

21. Ronco P, Allegri L, Brianti E et al (1989) Antigenic targets in epimembranous glomerulonephritis. Experimental data and potential application in human pathology. Appl Pathol 7(2):85-98

22. Ronco PM, Ardaillou N, Verroust P et al (1994) Pathophysiology of the podocyte: a target and a major player in glomerulonephritis. Adv Nephrol Necker Hosp 23:91-131

23. Nortier JL, Debiec H, Tournay Y et al (2006) Neonatal disease in neutral endopeptidase alloimmunization: lessons for immunological monitoring. Pediatr Nephrol 21(10):1399-1405

24. Simister NE (2003) Placental transport of immunoglobulin G. Vaccine 21(24):3365-3369

25. Ronco P, Debiec H, Guigonis V (2006) Mechanisms of disease: alloimmunization in renal diseases. Nat Clin Pract Nephrol 2(7):388-397

26. Debiec H, Ronco P (2007) Fetomaternal alloimmunization with antenatal glomerulopathies. Ann N Y Acad Sci 1110:559-566

27. Ronco P, Debiec H (2007) Podocyte antigens and glomerular disease. Nephron Exp Nephrol 107(2):e41-e46

28. Clark MR (1997) IgG effector mechanisms. Chem Immunol 65:88-110

29. Jefferis R, Pound J, Lund J et al (1994) Effector mechanisms activated by human IgG subclass antibodies: clinical and molecular aspects. Review article. Ann Biol Clin (Paris) 52(1):57-65

30. Ronco P, Debiec H (2005) Molecular pathomechanisms of membranous nephropathy: from Heymann nephritis to alloimmunization. J Am Soc Nephrol 16(5):1205-1213 
31. Bogdanos DP, Invernizzi P, Mackay IR et al (2008) Autoimmune liver serology: current diagnostic and clinical challenges. World $\mathbf{J}$ Gastroenterol 14(21):3374-3387

32. Metcalf JV, Mitchison HC, Palmer JM et al (1996) Natural history of early primary biliary cirrhosis. Lancet 348(9039):1399-1402

33. Neuberger J, Thomson R (1999) PBC and AMA-what is the connection? Hepatology 29(1):271-276

34. Liu H, Norman GL, Shums Z et al (2011) PBC screen: an IgG/ IgA dual isotype ELISA detecting multiple mitochondrial and nuclear autoantibodies specific for primary biliary cirrhosis. J Autoimmun 35(4):436-442

35. Rigopoulou EI, Bogdanos DP, Liaskos C et al (2007) Antimitochondrial antibody immunofluorescent titres correlate with the number and intensity of immunoblot-detected mitochondrial bands in patients with primary biliary cirrhosis. Clin Chim Acta 380(1-2):118-121

36. Melegh B, Skuta G, Pajor L et al (1998) Autoantibodies against subunits of pyruvate dehydrogenase and citrate synthase in a case of paediatric biliary cirrhosis. Gut 42(5):753-756

37. Zamfir O, Briaud I, Dubel L et al (1999) Anti-pyruvate dehydrogenase autoantibodies in extrahepatic disorders. J Hepatol 31(5):964-965

38. Gregorio GV, Portmann B, Mowat AP et al (1997) A 12-year-old girl with antimitochondrial antibody-positive autoimmune hepatitis. J Hepatol 27(4):751-754

39. Dahlan Y, Smith L, Simmonds D et al (2003) Pediatric-onset primary biliary cirrhosis. Gastroenterology 125(5):1476-1479

40. Tsuda M, Torgerson TR, Selmi C et al (2011) The spectrum of autoantibodies in IPEX syndrome is broad and includes antimitochondrial autoantibodies. J Autoimmun 35(3):265-268

41. Aoki CA, Roifman CM, Lian ZX et al (2006) IL-2 receptor alpha deficiency and features of primary biliary cirrhosis. J Autoimmun 27(1):50-53

42. Rigopoulou EI, Davies ET, Bogdanos DP et al (2007) Antimitochondrial antibodies of immunoglobulin G3 subclass are associated with a more severe disease course in primary biliary cirrhosis. Liver Int 27(9):1226-1231

43. Selmi C, Meda F, Kasangian A et al (2011) Experimental evidence on the immunopathogenesis of primary biliary cirrhosis. Cell Mol Immunol 7(1):1-10

44. Selmi C, Mackay IR, Gershwin ME (2007) The immunological milieu of the liver. Semin Liver Dis 27(2):129-139

45. Selmi C, Gershwin ME (2010) Autoantibodies in autoimmune liver disease: biomarkers versus epiphenomena. Gut 59(6): 712-713

46. Selmi C, Zuin M, Bowlus CL et al (2008) Anti-mitochondrial antibody-negative primary biliary cirrhosis. Clin Liver Dis 12(1):173-185, ix

47. Selmi C, Zuin M, Gershwin ME (2008) The unfinished business of primary biliary cirrhosis. J Hepatol 49(3):451-460

48. Vergani D, Bogdanos DP, Baum H (2004) Unusual suspects in primary biliary cirrhosis. Hepatology 39(1):38-41

49. Selmi C, Cocchi CA, Zuin M et al (2009) The chemical pathway to primary biliary cirrhosis. Clin Rev Allergy Immunol 36(1):23-29

50. Gershwin ME, Mackay IR (2008) The causes of primary biliary cirrhosis: convenient and inconvenient truths. Hepatology 47(2):737-745

51. Bogdanos D, Pusl T, Rust C et al (2008) Primary biliary cirrhosis following Lactobacillus vaccination for recurrent vaginitis. J Hepatol 49(3):466-473

52. Bogdanos DP, Baum H, Grasso A et al (2004) Microbial mimics are major targets of crossreactivity with human pyruvate dehydrogenase in primary biliary cirrhosis. J Hepatol 40(1):31-39

53. Bogdanos DP, Baum H, Gunsar F et al (2004) Extensive homology between the major immunodominant mitochondrial antigen in primary biliary cirrhosis and Helicobacter pylori does not lead to immunological cross-reactivity. Scand J Gastroenterol 39(10):981-987

54. Bogdanos DP, Baum H, Okamoto M et al (2005) Primary biliary cirrhosis is characterized by IgG3 antibodies cross-reactive with the major mitochondrial autoepitope and its Lactobacillus mimic. Hepatology 42(2):458-465

55. Bogdanos DP, Baum H, Sharma UC et al (2002) Antibodies against homologous microbial caseinolytic proteases $\mathrm{P}$ characterise primary biliary cirrhosis. J Hepatol 36(1):14-21

56. Bogdanos DP, Choudhuri K, Vergani D (2001) Molecular mimicry and autoimmune liver disease: virtuous intentions, malign consequences. Liver 21(4):225-232

57. Bogdanos DP, Dalekos GN (2008) Enzymes as target antigens of liver-specific autoimmunity: the case of cytochromes P450s. Curr Med Chem 15(22):2285-2292

58. Bogdanos DP, Koutsoumpas A, Baum H et al (2006) Borrelia Burgdorferi: a new self-mimicking trigger in primary biliary cirrhosis. Dig Liver Dis 38(10):781-782 (author reply 782-783)

59. Bogdanos DP, Mieli-Vergani G, Vergani D (2000) Virus, liver and autoimmunity. Dig Liver Dis 32(5):440-446

60. Bogdanos DP, Pares A, Baum H et al (2004) Disease-specific cross-reactivity between mimicking peptides of heat shock protein of Mycobacterium gordonae and dominant epitope of E2 subunit of pyruvate dehydrogenase is common in Spanish but not British patients with primary biliary cirrhosis. J Autoimmun 22(4):353-362

61. Bogdanos DP, Vergani D (2006) Origin of cross-reactive autoimmunity in primary biliary cirrhosis. Liver Int 26(6):633-635

62. Bogdanos DP, Vergani D (2009) Bacteria and primary biliary cirrhosis. Clin Rev Allergy Immunol 36(1):30-39

63. Koutsoumpas A, Mytilinaiou M, Polymeros D et al (2009) AntiHelicobacter pylori antibody responses specific for VacA do not trigger primary biliary cirrhosis-specific antimitochondrial antibodies. Eur J Gastroenterol Hepatol 21(10):1220

64. Van Norstrand MD, Malinchoc M, Lindor KD et al (1997) Quantitative measurement of autoantibodies to recombinant mitochondrial antigens in patients with primary biliary cirrhosis: relationship of levels of autoantibodies to disease progression. Hepatology 25(1):6-11

65. Mitchison HC, Bassendine MF, Hendrick A et al (1986) Positive antimitochondrial antibody but normal alkaline phosphatase: is this primary biliary cirrhosis? Hepatology 6(6):1279-1284

66. Kisand KE, Metskula K, Kisand KV et al (2001) The follow-up of asymptomatic persons with antibodies to pyruvate dehydrogenase in adult population samples. J Gastroenterol 36(4): $248-254$

67. Kita H, Lian ZX, Van de Water J et al (2002) Identification of HLA-A2-restricted CD8(+) cytotoxic T cell responses in primary biliary cirrhosis: $\mathrm{T}$ cell activation is augmented by immune complexes cross-presented by dendritic cells. J Exp Med 195(1):113-123

68. Lleo A, Selmi C, Invernizzi P et al (2009) Apotopes and the biliary specificity of primary biliary cirrhosis. Hepatology 49(3):871-879

69. Lleo A, Selmi C, Invernizzi P, Podda M et al (2008) The consequences of apoptosis in autoimmunity. J Autoimmun 31(3):257-262

70. Durand P, Debray D, Mandel R et al (2001) Acute liver failure in infancy: a 14-year experience of a pediatric liver transplantation center. J Pediatr 139(6):871-876

71. Kershisnik MM, Knisely AS, Sun CC et al (1992) Cytomegalovirus infection, fetal liver disease, and neonatal hemochromatosis. Hum Pathol 23(9): 1075-1080

72. Knisely AS (1988) Neonatal hemochromatosis. Am J Gastroenterol 83(11):1312-1313 
73. Knisely AS (1992) Neonatal hemochromatosis. Adv Pediatr 39:383-403

74. Knisely AS, Harford JB, Klausner RD et al (1989) Neonatal hemochromatosis. The regulation of transferrin-receptor and ferritin synthesis by iron in cultured fibroblastic-line cells. Am J Pathol 134(2):439-445

75. Knisely AS, Magid MS, Dische MR et al (1987) Neonatal hemochromatosis. Birth Defects Orig Artic Ser 23(1):75-102

76. Bonilla SF, Melin-Aldana H, Whitington PF (2009) Relationship of proximal renal tubular dysgenesis and fetal liver injury in neonatal hemochromatosis. Pediatr Res 67(2):188-193

77. Malladi P, Wang H, Pan X et al (2006) The pathogenesis of neonatal hemochromatosis involves maternal IGG alloantibody mediated complement-dependent hepatocytolysis and necrosis. Hepatology 44:56A
78. Pan X, Kelly S, Melin-Aldana H (2010) Novel mechanism of fetal hepatocyte injury in congenital alloimmune hepatitis involves the terminal complement cascade. Hepatology 51(6):2061-2068

79. Knisely AS, Vergani D (2010) "Neonatal hemochromatosis": a re-vision. Hepatology 51(6):1888-1890

80. Venkat-Raman N, Venkata-Krishnan RV, Howarth ES (2006) Successful pregnancy outcome following maternal intravenous immunoglobulin treatment in a woman with recurrent perinatal haemochromatosis. Prenat Diagn 26(13):1256-1259

81. Carrabin N, Cordier MP, Gaucherand P (2007) High-dose immunoglobulin during pregnancy for two patients with risk of recurrent neonatal haemochromatosis. J Gynecol Obstet Biol Reprod (Paris) 36(4):409-412 